\title{
Topological Properties for Harmonic $\tau$-Uniformly Convex Functions of Order $\rho$ Associated with Wanas Differential Operator
}

\section{Abbas Kareem Wanas}

\begin{abstract}
The purpose of the present paper is to establish some topological properties for a certain family of harmonic $\tau$-uniformly convex functions of order $\rho$ associated with Wanas differential operator defined in the open unit disk $U$.
\end{abstract}

\section{Introduction}

A continuous function $f=u+i v$ is a complex valued harmonic function in a complex domain $\mathbb{C}$, if both $u$ and $v$ are real harmonic in $\mathbb{C}$. In any simply connected domain $D \subset \mathbb{C}$, we can write $f=h+\bar{g}$, where $h$ and $g$ are analytic in $D$. We call $h$ the analytic part and $g$ the co-analytic part of $f$. A necessary and sufficient condition for $f$ to be locally univalent and sense-preserving in $D$ is that $\left|h^{\prime}(z)\right|>\left|g^{\prime}(z)\right|$ in $D$ (see Clunie and Sheil-Small [6]).

Denote by $\mathcal{H}$ the family of harmonic functions in the open unit disk $U=\{z \in \mathbb{C}$ : $|z|<1\}$. Let $S_{\mathcal{H}}$ indicate the family of functions $f=h+\bar{g} \in \mathcal{H}$ which are univalent and sense-preserving in the open unit disk $U$ and normalized by $f(0)=f_{z}(0)-1=0$. Each $f \in S_{\mathcal{H}}$ can be expressed as

$$
f(z)=h(z)+\overline{g(z)}
$$

where

$$
h(z)=z+\sum_{n=2}^{\infty} a_{n} z^{n}, \quad g(z)=\sum_{n=2}^{\infty} b_{n} z^{n} .
$$

Received: May 19, 2020; Accepted: June 17, 2020

2010 Mathematics Subject Classification: 30C55, 30C45.

Keywords and phrases: harmonic function, uniformly convex function, extreme points, closed convex hull, compact set. 
Also note that $\mathcal{H}$ reduces to the family $\mathcal{A}$ of analytic functions in $U$ if co-analytic part of $f$ is identically zero.

A function $f \in S_{\mathcal{H}}$ is said to be harmonic starlike in $U(r)=\{z \in \mathbb{C}:|z|<r\}$, if (see [11])

$$
\frac{\partial}{\partial t}\left(\arg \left(f\left(r e^{i t}\right)\right)\right)=\operatorname{Re}\left\{\frac{z h^{\prime}(z)-\overline{z g^{\prime}(z)}}{h(z)+\overline{g(z)}}\right\}>0, \quad(0 \leq t \leq 2 \pi)
$$

i.e., $f$ maps the circle $\partial U(r)$ onto a closed curve that is starlike with respect to the origin.

We consider the usual topology on $\mathcal{H}$ defined by a metric in which a sequence $\left\{f_{n}\right\}$ in $\mathcal{H}$ converges to $f$ if and only if it converges to $f$ uniformly on each compact subset of $U$. It follows from the theorems of Weierstrass and Montel that this topological space is complete.

Let $\mathcal{M}$ be a sub-family of the set $\mathcal{H}$. A function $f \in \mathcal{M}$ is called an extreme point of $\mathcal{M}$ if the condition $f=\lambda f_{1}+(1-\lambda) f_{2}\left(f_{1}, f_{2} \in \mathcal{M} ; 0<\lambda<1\right)$ implies $f_{1}=f_{2}=f$.

We denote by $E \mathcal{M}$ the set of all extreme points of $\mathcal{M}$. It is clear that $E \mathcal{M} \subset \mathcal{M}$.

A family $\mathcal{M}$ is locally uniformly bounded if for each $r(0<r<1)$, there is a real constant $V=V(r)$ so that $|f(z)| \leq V(f \in \mathcal{M} ;|z| \leq r)$.

A family $\mathcal{M}$ is convex if $\gamma f_{1}+(1-\gamma) f_{2} \in \mathcal{M}\left(f_{1}, f_{2} \in \mathcal{M} ; 0 \leq \gamma \leq 1\right)$.

Moreover, we define the closed convex hull of $\mathcal{M}$ as the intersection of all closed convex subsets of $\mathcal{H}$ (with respect to the topology of locally uniform convergence) that contain $\mathcal{M}$. We denote the closed convex hull of $\mathcal{M}$ by $\overline{c o} \mathcal{M}$.

A real-valued functional $F: \mathcal{H} \rightarrow \mathbb{R}$ is called convex on a convex family $\mathcal{M} \subset \mathcal{H}$ if

$$
F\left(\gamma f_{1}+(1-\gamma) f_{2}\right) \leq \gamma F\left(f_{1}\right)+(1-\gamma) F\left(f_{2}\right) \quad\left(f_{1}, f_{2} \in \mathcal{M} ; 0 \leq \gamma \leq 1\right) .
$$

For $\alpha \in \mathbb{R}, \beta \geq 0$ with $\alpha+\beta>0, m, q \in \mathbb{N}_{0}=\mathbb{N} \cup\{0\}$ and for analytic part $h \in$ $\mathcal{A}$, Wanas [15] introduced an operator (so-called Wanas operator) $W_{\alpha, \beta}^{k, q}: \mathcal{A} \rightarrow \mathcal{A}$, defined by

$$
W_{\alpha, \beta}^{k, q} h(z)=z+\sum_{n=2}^{\infty}\left[\Phi_{n}(k, \alpha, \beta)\right]^{q} a_{n} z^{n}
$$


where

$$
\Phi_{n}(k, \alpha, \beta)=\sum_{m=1}^{k}\left(\begin{array}{l}
k \\
m
\end{array}\right)(-1)^{m+1}\left(\frac{\alpha^{m}+n \beta^{m}}{\alpha^{m}+\beta^{m}}\right) .
$$

Special cases of this operator can be found in $[1,2,3,4,5,8,10,12,13,14]$. For more details see [16].

Now, we extended this operator on the family of harmonic functions. For $f=h+$ $\bar{g} \in \mathcal{H}$, we define the Wanas differential operator $W_{\alpha, \beta}^{k, q}: \mathcal{H} \rightarrow \mathcal{H}$ as follows:

$$
W_{\alpha, \beta}^{k, q} f(z)=W_{\alpha, \beta}^{k, q} h(z)+(-1)^{q} \overline{W_{\alpha, \beta}^{k, q} g(z)},
$$

where $W_{\alpha, \beta}^{k, q} h(z)$ is defined by (1.2) and

$$
W_{\alpha, \beta}^{k, q} g(z)=\sum_{n=2}^{\infty}\left[\Phi_{n}(k, \alpha, \beta)\right]^{q} b_{n} z^{n} .
$$

We denote by $W S_{\mathcal{H}}(\rho, \tau, \alpha, \beta, k, q)$ the family of all functions of the form (1.1) that satisfy the condition:

$$
\operatorname{Re}\left\{\frac{z\left(W_{\alpha, \beta}^{k, q} h(z)+(-1)^{q} \overline{W_{\alpha, \beta}^{k, q} g(z)}\right)^{\prime \prime}}{\left(W_{\alpha, \beta}^{k, q} h(z)+(-1)^{q} \overline{W_{\alpha, \beta}^{k, q} g(z)}\right)^{\prime}}+1\right\}>\tau\left|\frac{z\left(W_{\alpha, \beta}^{k, q} h(z)+(-1)^{q} \overline{W_{\alpha, \beta}^{k, q} g(z)}\right)^{\prime \prime}}{\left(W_{\alpha, \beta}^{k, q} h(z)+(-1)^{q} \overline{W_{\alpha, \beta}^{k, q} g(z)}\right)^{\prime}}\right|+\rho,
$$

where $0 \leq \rho<1 ; \tau \geq 0$ and $z \in U$.

Also denote by $T_{\mathcal{H}}$ the sub-family of $S_{\mathcal{H}}$ containing of all functions $f=h+\bar{g}$, where $h$ and $g$ are given by

$$
h(z)=z-\sum_{n=2}^{\infty}\left|a_{n}\right| z^{n}, \quad g(z)=(-1)^{q} \sum_{n=2}^{\infty}\left|b_{n}\right| z^{n} .
$$

It is easily verified that if $f \in T_{\mathcal{H}}$, we also have

$$
W_{\alpha, \beta}^{k, q} f(z)=z-\sum_{n=2}^{\infty}\left|\Phi_{n}(k, \alpha, \beta)\right|^{q}\left|a_{n}\right| z^{n}+(-1)^{q} \sum_{n=2}^{\infty}\left|\Phi_{n}(k, \alpha, \beta)\right|^{q}\left|b_{n}\right|(\bar{z})^{n} .
$$

Moreover, let $W T_{\mathcal{H}}(\rho, \tau, \alpha, \beta, k, q)$ be the sub-family of $W S_{\mathcal{H}}(\rho, \tau, \alpha, \beta, k, q)$, where

$$
W T_{\mathcal{H}}(\rho, \tau, \alpha, \beta, k, q)=T_{\mathcal{H}} \cap W S_{\mathcal{H}}(\rho, \tau, \alpha, \beta, k, q) .
$$


We now recall the following lemmas that will be used to prove our main results.

Lemma 1.1 [7]. Let $\mathcal{M}$ be a nonempty compact convex subset of the family $\mathcal{H}$ and $F: \mathcal{H} \rightarrow \mathbb{R}$ be a real-valued, continuous and convex functional on $\mathcal{M}$. Then

$$
\max \{F(f): f \in \mathcal{M}\}=\max \{F(f): f \in E \mathcal{M}\} .
$$

Lemma 1.2 [9]. A family $\mathcal{M} \subset \mathcal{H}$ is compact if and only if $\mathcal{M}$ is closed and locally uniformly bounded.

\section{A Set of Main Results}

In the first theorem, we determine the sufficient condition for $f=h+\bar{g}$ to be in the family $W S_{\mathcal{H}}(\rho, \tau, \alpha, \beta, k, q)$.

Theorem 2.1. Let $f=h+\bar{g}$ with $h$ and $g$ are given by (1.1). If

$$
\sum_{n=2}^{\infty} n(n-\rho+(n-1) \tau)\left|\Phi_{n}(k, \alpha, \beta)\right|^{q}\left(\left|a_{n}\right|+\left|b_{n}\right|\right) \leq 1-\rho,
$$

where $0 \leq \rho<1, \tau \geq 0$, then $f$ is harmonic univalent in $U$ and $f \in W S_{\mathcal{H}}(\rho, \tau, \alpha, \beta, k, q)$.

Proof. For proving $f \in W S_{\mathcal{H}}(\rho, \tau, \alpha, \beta, k, q)$, we must show that (1.3) holds true. Using the fact that $\operatorname{Re}\{w\} \geq \tau$ if and only if $|1-\tau+w| \geq|1+\tau-w|$, it is suffices to show that

$$
\operatorname{Re}\left\{\left(\frac{z\left(W_{\alpha, \beta}^{k, q} h(z)+(-1)^{q} \overline{W_{\alpha, \beta}^{k, q} g(z)}\right)^{\prime \prime}}{\left(W_{\alpha, \beta}^{k, q} h(z)+(-1)^{q} \overline{W_{\alpha, \beta}^{k, q} g(z)}\right)^{\prime}}+1\right)\left(1+\tau e^{i \theta}\right)-\tau e^{i \theta}\right\}>\rho \quad(-\pi \leq \theta \leq \pi),
$$

or equivalently

$$
\begin{gathered}
\operatorname{Re}\left\{\frac{\left(1+\tau e^{i \theta}\right)\left(z\left(W_{\alpha, \beta}^{k, q} h(z)+(-1)^{q} \overline{W_{\alpha, \beta}^{k, q} g(z)}\right)^{\prime \prime}+\left(W_{\alpha, \beta}^{k, q} h(z)+(-1)^{q} \overline{W_{\alpha, \beta}^{k, q} g(z)}\right)^{\prime}\right)}{\left(W_{\alpha, \beta}^{k, q} h(z)+(-1)^{q} \overline{W_{\alpha, \beta}^{k, q} g(z)}\right)^{\prime}}\right. \\
\left.-\frac{\tau e^{i \theta}\left(W_{\alpha, \beta}^{k, q} h(z)+(-1)^{q} \overline{W_{\alpha, \beta}^{k, q} g(z)}\right)^{\prime}}{\left(W_{\alpha, \beta}^{k, q} h(z)+(-1)^{q} \overline{W_{\alpha, \beta}^{k, q} g(z)}\right)^{\prime}}\right\}>\rho . \quad \text { (2.2) }
\end{gathered}
$$


If we put

$$
\begin{aligned}
A(z)=\left(1+\tau e^{i \theta}\right)\left(z\left(W_{\alpha, \beta}^{k, q} h(z)+(-1)^{q} \overline{W_{\alpha, \beta}^{k, q} g(z)}\right)^{\prime \prime}\right. & \\
+ & \left.\left(W_{\alpha, \beta}^{k, q} h(z)+(-1)^{q} \overline{W_{\alpha, \beta}^{k, q} g(z)}\right)^{\prime}\right) \\
& -\tau e^{i \theta}\left(W_{\alpha, \beta}^{k, q} h(z)+(-1)^{q} \overline{W_{\alpha, \beta}^{k, q} g(z)}\right)^{\prime}
\end{aligned}
$$

and

$$
B(z)=\left(W_{\alpha, \beta}^{k, q} h(z)+(-1)^{q} \overline{W_{\alpha, \beta}^{k, q} g(z)}\right)^{\prime} .
$$

We only need to prove that

$$
|A(z)+(1-\rho) B(z)|-|A(z)-(1+\rho) B(z)| \geq 0 .
$$

But

$$
\begin{aligned}
& |A(z)+(1-\rho) B(z)| \\
= & \mid\left(1+\tau e^{i \theta}\right)\left(\sum_{n=2}^{\infty} n(n-1)\left[\Phi_{n}(k, \alpha, \beta)\right]^{q} a_{n} z^{n-1}+(-1)^{q} \sum_{n=2}^{\infty} n(n-1)\left[\Phi_{n}(k, \alpha, \beta)\right]^{q} \overline{b_{n}}(\bar{z})^{n-1}\right. \\
& \left.+1+\sum_{n=2}^{\infty} n\left[\Phi_{n}(k, \alpha, \beta)\right]^{q} a_{n} z^{n-1}+(-1)^{q} \sum_{n=2}^{\infty} n\left[\Phi_{n}(k, \alpha, \beta)\right]^{q} \overline{b_{n}}(\bar{z})^{n-1}\right) \\
& -\tau e^{i \theta}\left(1+\sum_{n=2}^{\infty} n\left[\Phi_{n}(k, \alpha, \beta)\right]^{q} a_{n} z^{n-1}+(-1)^{q} \sum_{n=2}^{\infty} n\left[\Phi_{n}(k, \alpha, \beta)\right]^{q} \overline{b_{n}}(\bar{z})^{n-1}\right) \\
& +(1-\rho)\left(1+\sum_{n=2}^{\infty} n\left[\Phi_{n}(k, \alpha, \beta)\right]^{q} a_{n} z^{n-1}+(-1)^{q} \sum_{n=2}^{\infty} n\left[\Phi_{n}(k, \alpha, \beta)\right]^{q} \overline{b_{n}}(\bar{z})^{n-1}\right) \mid \\
= & \mid(2-\rho)+\sum_{n=2}^{\infty} n\left(n+1-\rho+(n-1) \tau e^{i \theta}\right)\left[\Phi_{n}(k, \alpha, \beta)\right]^{q} a_{n} z^{n-1} \\
& +(-1)^{q} \sum_{n=2}^{\infty} n\left(n+1-\rho+(n-1) \tau e^{i \theta}\right)\left[\Phi_{n}(k, \alpha, \beta)\right]^{q} \overline{b_{n}}(\bar{z})^{n-1} \mid .
\end{aligned}
$$


Also

$$
\begin{aligned}
& |A(z)-(1+\rho) B(z)| \\
= & \mid\left(1+\rho e^{i \theta}\right)\left(\sum_{n=2}^{\infty} n(n-1)\left[\Phi_{n}(k, \alpha, \beta)\right]^{q} a_{n} z^{n-1}+(-1)^{q} \sum_{n=2}^{\infty} n(n-1)\left[\Phi_{n}(k, \alpha, \beta)\right]^{q} \overline{b_{n}}(\bar{z})^{n-1}\right. \\
& \left.+1+\sum_{n=2}^{\infty} n\left[\Phi_{n}(k, \alpha, \beta)\right]^{q} a_{n} z^{n-1}+(-1)^{q} \sum_{n=2}^{\infty} n\left[\Phi_{n}(k, \alpha, \beta)\right]^{q} \overline{b_{n}}(\bar{z})^{n-1}\right) \\
& -\tau e^{i \theta}\left(1+\sum_{n=2}^{\infty} n\left[\Phi_{n}(k, \alpha, \beta)\right]^{q} a_{n} z^{n-1}+(-1)^{q} \sum_{n=2}^{\infty} n\left[\Phi_{n}(k, \alpha, \beta)\right]^{q} \overline{b_{n}}(\bar{z})^{n-1}\right) \\
& -(1+\rho)\left(1+\sum_{n=2}^{\infty} n\left[\Phi_{n}(k, \alpha, \beta)\right]^{q} a_{n} z^{n-1}+(-1)^{q} \sum_{n=2}^{\infty} n\left[\Phi_{n}(k, \alpha, \beta)\right]^{q} \overline{b_{n}}(\bar{z})^{n-1}\right) \mid \\
= & \mid-\rho+\sum_{n=2}^{\infty} n\left(n-1-\rho+(n-1) \tau e^{i \theta}\right)\left[\Phi_{n}(k, \alpha, \beta)\right]^{q} a_{n} z^{n-1} \\
& +(-1)^{q} \sum_{n=2}^{\infty} n\left(n-1-\rho+(n-1) \tau e^{i \theta}\right)\left[\Phi_{n}(k, \alpha, \beta)\right]^{q} \overline{b_{n}}(\bar{z})^{n-1} \mid .
\end{aligned}
$$

Then

$$
\begin{aligned}
& |A(z)+(1-\rho) B(z)|-|A(z)-(1+\rho) B(z)| \\
\geq & 2(1-\rho)-\sum_{n=2}^{\infty} 2 n(n-\rho+(n-1) \tau)\left|\Phi_{n}(k, \alpha, \beta)\right|^{q}\left|a_{n}\right||z|^{n-1} \\
& -\sum_{n=2}^{\infty} 2 n(n-\rho+(n-1) \tau)\left|\Phi_{n}(k, \alpha, \beta)\right|^{q}\left|b_{n}\right||z|^{n-1} \\
> & 2\left\{(1-\rho)-\sum_{n=2}^{\infty} n(n-\rho+(n-1) \tau)\left|\Phi_{n}(k, \alpha, \beta)\right|^{q}\left(\left|a_{n}\right|+\left|b_{n}\right|\right)\right\}>0 .
\end{aligned}
$$

The harmonic univalent function

$$
f(z)=z+\sum_{n=2}^{\infty} \frac{x_{n}}{n(n-\rho+(n-1) \tau)\left|\Phi_{n}(k, \alpha, \beta)\right|^{q}} z^{n}
$$




$$
+\sum_{n=2}^{\infty} \frac{\bar{y}_{n}}{n(n-\rho+(n-1) \tau)\left|\Phi_{n}(k, \alpha, \beta)\right|^{q}}(\bar{z})^{n}
$$

where

$$
\sum_{n=2}^{\infty}\left|x_{n}\right|+\sum_{n=1}^{\infty}\left|y_{n}\right|=1-\rho
$$

shows that the coefficient bound given by (2.1) is sharp.

The functions of the form (2.3) are in the family $W S_{\mathcal{H}}(\rho, \tau, \alpha, \beta, k, q)$, because

$$
\begin{aligned}
& \sum_{n=2}^{\infty} n(n-\rho+(n-1) \tau)\left|\Phi_{n}(k, \alpha, \beta)\right|^{q} \frac{\left|x_{n}\right|}{n(n-\rho+(n-1) \tau)\left|\Phi_{n}(k, \alpha, \beta)\right|^{q}} \\
& +\sum_{n=2}^{\infty} n(n-\rho+(n-1) \tau)\left|\Phi_{n}(k, \alpha, \beta)\right|^{q} \frac{\left|y_{n}\right|}{n(n-\rho+(n-1) \tau)\left|\Phi_{n}(k, \alpha, \beta)\right|^{q}} \\
= & \sum_{n=2}^{\infty}\left|x_{n}\right|+\sum_{n=1}^{\infty}\left|y_{n}\right|=1-\rho .
\end{aligned}
$$

The restriction placed in Theorem 2.1 on the moduli of the coefficients of $f=h+\bar{g}$ enables us to conclude for arbitrary rotation of the coefficients of $f$ that the resulting functions would still be harmonic univalent and $f \in W S_{\mathcal{H}}(\rho, \tau, \alpha, \beta, k, q)$.

The next theorem shows that condition (2.1) is also the sufficient condition for functions $f \in T_{\mathcal{H}}$ to be in the family $W T_{\mathcal{H}}(\rho, \tau, \alpha, \beta, k, q)$.

Theorem 2.2. Let $f \in T_{\mathcal{H}}$ be a function of the form (1.4). Then $f \in W T_{\mathcal{H}}(\rho, \tau, \alpha, \beta, k, q)$ if and only if condition (2.1) holds true.

Proof. In the light of Theorem 2.1, we need only to prove that each function $f \in$ $W T_{\mathcal{H}}(\rho, \tau, \alpha, \beta, k, q)$ satisfies coefficient inequality (2.1). If $f \in W T_{\mathcal{H}}(\rho, \tau, \alpha, \beta, k, q)$, then by (1.3), we have

$$
\operatorname{Re}\left\{\left(\frac{z\left(W_{\alpha, \beta}^{k, q} h(z)+(-1)^{q} \overline{W_{\alpha, \beta}^{k, q} g(z)}\right)^{\prime \prime}}{\left(W_{\alpha, \beta}^{k, q} h(z)+(-1)^{q} \overline{W_{\alpha, \beta}^{k, q} g(z)}\right)^{\prime}}+1\right)\left(1+\tau e^{i \theta}\right)-\tau e^{i \theta}\right\}>\rho(-\pi \leq \theta \leq \pi) .
$$


This is equivalent to

$$
\begin{aligned}
& \operatorname{Re}\left\{\frac{\left(1+\tau e^{i \theta}\right)\left(z\left(W_{\alpha, \beta}^{k, q} h(z)+(-1)^{q} \overline{W_{\alpha, \beta}^{k, q} g(z)}\right)^{\prime \prime}+\left(W_{\alpha, \beta}^{k, q} h(z)+(-1)^{q} \overline{W_{\alpha, \beta}^{k, q} g(z)}\right)^{\prime}\right)}{\left(W_{\alpha, \beta}^{k, q} h(z)+(-1)^{q} \overline{W_{\alpha, \beta}^{k, q} g(z)}\right)^{\prime}}\right. \\
& \left.-\frac{\tau e^{i \theta}\left(W_{\alpha, \beta}^{k, q} h(z)+(-1)^{q} \overline{W_{\alpha, \beta}^{k, q} g(z)}\right)^{\prime}}{\left(W_{\alpha, \beta}^{k, q} h(z)+(-1)^{q} \overline{W_{\alpha, \beta}^{k, q} g(z)}\right)^{\prime}}\right\} \\
& =\operatorname{Re} \frac{(1-\rho)-\sum_{n=2}^{\infty} n\left(n-\rho+(n-1) \tau e^{i \theta}\right)\left|\Phi_{n}(k, \alpha, \beta)\right|^{q}\left|a_{n}\right| z^{n-1}-}{1-\sum_{n=2}^{\infty} n\left|\Phi_{n}(k, \alpha, \beta)\right|^{q}\left|a_{n}\right| z^{n-1}-\sum_{n=2}^{\infty} n\left|\Phi_{n}(k, \alpha, \beta)\right|^{q}\left|b_{n}\right|(\bar{z})^{n-1}} \\
& \left.-\frac{1-\sum_{n=2}^{\infty} n\left|\Phi_{n}(k, \alpha, \beta)\right|^{q}\left|a_{n}\right| z^{n-1}-\sum_{n=2}^{\infty} n\left|\Phi_{n}(k, \alpha, \beta)\right|^{q}\left|b_{n}\right|(\bar{z})^{n-1}}{\sum_{n=1}^{\infty} n\left(n-\rho+(n-1) \tau e^{i \theta}\right)\left|\Phi_{n}(k, \alpha, \beta)\right|^{q}\left|b_{n}\right|(\bar{z})^{n-1}}\right\} \geq 0 .
\end{aligned}
$$

The above required condition (2.4) must hold for all values of $z$ in $U$. Upon choosing the values of $z$ on the positive real axis where $0 \leq z=r<1$, we must have

$$
\operatorname{Re}\left\{\frac{(1-\rho)-\left[\sum_{n=2}^{\infty} n(n-\rho)\left|\Phi_{n}(k, \alpha, \beta)\right|^{q}\left|a_{n}\right| r^{n-1}+\sum_{n=1}^{\infty} n(n-\rho)\left|\Phi_{n}(k, \alpha, \beta)\right|^{q}\left|b_{n}\right| r^{n-1}\right]}{1-\sum_{n=2}^{\infty} n\left|\Phi_{n}(k, \alpha, \beta)\right|^{q}\left|a_{n}\right| r^{n-1}-\sum_{n=1}^{\infty} n\left|\Phi_{n}(k, \alpha, \beta)\right|^{q}\left|b_{n}\right| r^{n-1}}\right.
$$

$$
\left.-\frac{\tau e^{i \theta}\left[\sum_{n=2}^{\infty} n(n-1)\left|\Phi_{n}(k, \alpha, \beta)\right|^{q}\left|a_{n}\right| r^{n-1}+\sum_{n=2}^{\infty} n(n-1)\left|\Phi_{n}(k, \alpha, \beta)\right|^{q}\left|b_{n}\right| r^{n-1}\right]}{1-\sum_{n=2}^{\infty} n\left|\Phi_{n}(k, \alpha, \beta)\right|^{q}\left|a_{n}\right| r^{n-1}-\sum_{n=2}^{\infty} n\left|\Phi_{n}(k, \alpha, \beta)\right|^{q}\left|b_{n}\right| r^{n-1}}\right\} \geq 0 .
$$

Since $\operatorname{Re}\left(-e^{i \theta}\right) \geq-\left|e^{i \theta}\right|=-1$, and let $r \rightarrow 1^{-}$, the above inequality reduces to 


$$
\begin{gathered}
1-\rho-\sum_{n=2}^{\infty} n(n-\rho+(n-1) \tau)\left|\Phi_{n}(k, \alpha, \beta)\right|^{q}\left|a_{n}\right| \\
1-\sum_{n=2}^{\infty} n\left|\Phi_{n}(k, \alpha, \beta)\right|^{q}\left|a_{n}\right|-\sum_{n=2}^{\infty} n\left|\Phi_{n}(k, \alpha, \beta)\right|^{q}\left|b_{n}\right| \\
\frac{\sum_{n=1}^{\infty} n(n-\rho+(n-1) \tau)\left|\Phi_{n}(k, \alpha, \beta)\right|^{q}\left|b_{n}\right|}{1-\sum_{n=2}^{\infty} n\left|\Phi_{n}(k, \alpha, \beta)\right|^{q}\left|a_{n}\right|-\sum_{n=2}^{\infty} n\left|\Phi_{n}(k, \alpha, \beta)\right|^{q}\left|b_{n}\right|}
\end{gathered}
$$

This gives coefficient inequality (2.1), so the proof is complete.

Theorem 2.3. The family $W T_{\mathcal{H}}(\rho, \tau, \alpha, \beta, k, q)$ is a convex and compact subset of $\mathcal{H}$.

Proof. Let $0 \leq \gamma \leq 1$ and $f_{1}, f_{2} \in W T_{\mathcal{H}}(\rho, \tau, \alpha, \beta, k, q)$ be functions of the form:

$$
f_{j}(z)=\sum_{\ell=0}^{\infty}\left(a_{j, \ell} z^{\ell}+\overline{b_{j, \ell} z^{\ell}}\right) \quad(z \in U, j \in\{1,2\}) .
$$

Since

$$
\begin{aligned}
& \gamma f_{1}(z)+(1-\gamma) f_{2}(z) \\
= & z-\sum_{n=2}^{\infty}\left(\gamma\left|a_{1, n}\right|+(1-\gamma)\left|a_{2, n}\right|\right) z^{n}-(-1)^{q}\left(\gamma\left|b_{1, n}\right|+(1-\gamma)\left|b_{2, n}\right|\right)(\bar{z})^{n} .
\end{aligned}
$$

Thus by Theorem 2.2, we have

$$
\begin{aligned}
& \begin{array}{l}
\sum_{n=2}^{\infty} n(n-\rho+ \\
\quad(n-1) \tau)\left|\Phi_{n}(k, \alpha, \beta)\right|^{q}\left(\left(\gamma\left|a_{1, n}\right|+(1-\gamma)\left|a_{2, n}\right|\right)\right. \\
\left.\quad+\left(\gamma\left|b_{1, n}\right|+(1-\gamma)\left|b_{2, n}\right|\right)\right)
\end{array} \\
& =\gamma \sum_{n=2}^{\infty} n(n-\rho+(n-1) \tau)\left|\Phi_{n}(k, \alpha, \beta)\right|^{q}\left(\left|a_{1, n}\right|+\left|b_{1, n}\right|\right) \\
& \quad+(1-\gamma) \sum_{n=2}^{\infty} n(n-\rho+(n-1) \tau)\left|\Phi_{n}(k, \alpha, \beta)\right|^{q}\left(\left|a_{2, n}\right|+\left|b_{2, n}\right|\right) \\
& \leq \gamma(1-\rho)+(1-\gamma)(1-\rho)=1-\rho .
\end{aligned}
$$


Therefore

$$
\gamma f_{1}(z)+(1-\gamma) f_{2}(z) \in W T_{\mathcal{H}}(\rho, \tau, \alpha, \beta, k, q)
$$

Hence the family $W T_{\mathcal{H}}(\rho, \tau, \alpha, \beta, k, q)$ is convex.

Furthermore, for $f \in W T_{\mathcal{H}}(\rho, \tau, \alpha, \beta, k, q),|z| \leq r, 0<r<1$, we have

$$
\begin{aligned}
|f(z)| & \leq r+\sum_{n=2}^{\infty}\left(\left|a_{n}\right|+\left|b_{n}\right|\right) \\
& <r+\sum_{n=2}^{\infty} n(n-\rho+(n-1) \tau)\left|\Phi_{n}(k, \alpha, \beta)\right|^{q}\left(\left|a_{n}\right|+\left|b_{n}\right|\right) \\
& \leq r+1-\rho .
\end{aligned}
$$

Then, we conclude that the family $W T_{\mathcal{H}}(\rho, \tau, \alpha, \beta, k, q)$ is locally uniformly bounded. By Lemma 1.2, we only need to show that it is closed, i.e., if $f_{j} \in W T_{\mathcal{H}}(\rho, \tau, \alpha, \beta, k, q)(j \in$ $\mathbb{N})$ and $f_{j} \rightarrow f$, then $f \in W T_{\mathcal{H}}(\rho, \tau, \alpha, \beta, k, q)$. Let $f_{j}$ and $f$ be given by (1.2) and (1.1), respectively. In view of Theorem 2.2 , we find that

$$
\sum_{n=2}^{\infty} n(n-\rho+(n-1) \tau)\left|\Phi_{n}(k, \alpha, \beta)\right|^{q}\left(\left|a_{j, n}\right|+\left|b_{j, n}\right|\right) \leq 1-\rho \quad(j \in \mathbb{N}) .
$$

Since $f_{j} \rightarrow f$, we conclude that $\left|a_{j, n}\right| \rightarrow\left|a_{n}\right|$ and $\left|b_{j, n}\right| \rightarrow\left|b_{n}\right|$ as $j \rightarrow \infty(j \in \mathbb{N})$. The sequence of partial sums $\left\{S_{n}\right\}$ associated with the series

$$
\sum_{n=2}^{\infty} n(n-\rho+(n-1) \tau)\left|\Phi_{n}(k, \alpha, \beta)\right|^{q}\left(\left|a_{n}\right|+\left|b_{n}\right|\right)
$$

is a non-decreasing sequence.

Furthermore, by (2.6) it is bounded by $1-\rho$. Thus, the sequence $\left\{S_{n}\right\}$ is convergent and

$$
\sum_{n=2}^{\infty} n(n-\rho+(n-1) \tau)\left|\Phi_{n}(k, \alpha, \beta)\right|^{q}\left(\left|a_{n}\right|+\left|b_{n}\right|\right)=\lim _{n \rightarrow \infty} S_{n} \leq 1-\rho .
$$

Hence $f \in W T_{\mathcal{H}}(\rho, \tau, \alpha, \beta, k, q)$ and this completes the proof. 
Theorem 2.4. $E W T_{\mathcal{H}}(\rho, \tau, \alpha, \beta, k, q)=\left\{h_{n}: n \in \mathbb{N}\right\} \cup\left\{g_{n}: n \in \mathbb{N}_{2}\right\}$, where

$$
\begin{aligned}
h_{1}(z)=z, \quad h_{n}(z) & =z-\frac{1-\rho}{n(n-\rho+(n-1) \tau)\left|\Phi_{n}(k, \alpha, \beta)\right|^{q}} z^{n}, \\
g_{n}(z) & =z+\frac{1-\rho}{n(n-\rho+(n-1) \tau)\left|\Phi_{n}(k, \alpha, \beta)\right|^{q}}(\bar{z})^{n} .
\end{aligned}
$$

Proof. Assume that $0<\lambda<1$ and $g_{n}=\lambda f_{1}+(1-\lambda) f_{2}$, where $f_{1}, f_{2} \in$ $W T_{\mathcal{H}}(\rho, \tau, \alpha, \beta, k, q)$ are functions of the form (2.5). Then by (2.1), we obtain

$$
\left|b_{1, n}\right|=\left|b_{2, n}\right|=\frac{1-\rho}{n(n-\rho+(n-1) \tau)\left|\Phi_{n}(k, \alpha, \beta)\right|^{q}}
$$

and $a_{1, j}=a_{2, j}=0$ for $j \in \mathbb{N}_{2}$ and $b_{1, j}=b_{2, j}=0$ for $j \in \mathbb{N}_{2} \backslash\{n\}$. Then we have $g_{n}=f_{1}=f_{2}$ and hence $g_{n} \in W T_{\mathcal{H}}(\rho, \tau, \alpha, \beta, k, q)$. Similarly, we prove that the functions $h_{n}$ of the form (2.7) are the extreme points of the family $W T_{\mathcal{H}}(\rho, \tau, \alpha, \beta, k, q)$. Assume that $f \in \operatorname{EWT}_{\mathcal{H}}(\rho, \tau, \alpha, \beta, k, q)$ and $f$ is not of the form (2.7), then there are $j \in \mathbb{N}_{2}$ such that

or

$$
0<\left|a_{j}\right|<\frac{1-\rho}{j(j-\rho+(j-1) \tau)\left|\Phi_{j}(k, \alpha, \beta)\right|^{q}}
$$

$$
0<\left|b_{j}\right|<\frac{1-\rho}{j(j-\rho+(j-1) \tau)\left|\Phi_{j}(k, \alpha, \beta)\right|^{q}}
$$

If

$$
0<\left|a_{j}\right|<\frac{1-\rho}{j(j-\rho+(j-1) \tau)\left|\Phi_{j}(k, \alpha, \beta)\right|^{q}}
$$

then, we taking

$$
\lambda=\frac{j(j-\rho+(j-1) \tau)\left|\Phi_{j}(k, \alpha, \beta)\right|^{q}}{1-\rho}\left|a_{j}\right| \quad \text { and } \quad \psi=\frac{1}{1-\lambda}\left(f-\lambda h_{j}\right) .
$$

We note that $0<\lambda<1, \quad h_{j} \neq \psi$ and $f=\lambda h_{j}+(1-\lambda) \psi$. Therefore, $f \notin$ $E W T_{\mathcal{H}}(\rho, \tau, \alpha, \beta, k, q)$.

If

$$
0<\left|b_{j}\right|<\frac{1-\rho}{j(j-\rho+(j-1) \tau)\left|\Phi_{j}(k, \alpha, \beta)\right|^{q}},
$$


then, we taking

$$
\lambda=\frac{j(j-\rho+(j-1) \tau)\left|\Phi_{j}(k, \alpha, \beta)\right|^{q}}{1-\rho}\left|b_{j}\right| \quad \text { and } \quad \phi=\frac{1}{1-\lambda}\left(f-\lambda g_{j}\right) .
$$

We note that $0<\lambda<1, \quad g_{j} \neq \phi \quad$ and $f=\lambda g_{j}+(1-\lambda) \phi$. Therefore, $f \notin E W T_{\mathcal{H}}(\rho, \tau, \alpha, \beta, k, q)$.

Remark 2.1. If the family $\mathcal{M}=\left\{f_{n} \in \mathcal{H}: n \in \mathbb{N}\right\}$ is locally uniformly bounded, then

$$
\overline{C O} \mathcal{M}=\left\{\sum_{n=1}^{\infty} \gamma_{n} f_{n}: \sum_{n=1}^{\infty} \gamma_{n}=1, \gamma_{n} \geq 0(n \in \mathbb{N})\right\} .
$$

Corollary 2.1. Let $h_{n}$ and $g_{n}$ be defined by (2.7). Then

$$
W T_{\mathcal{H}}(\rho, \tau, \alpha, \beta, k, q)=\left\{\sum_{n=1}^{\infty}\left(\gamma_{n} h_{n}+\mu_{n} g_{n}\right): \sum_{n=1}^{\infty}\left(\gamma_{n}+\mu_{n}\right)=1, \mu_{1}=0, \gamma_{n}, \mu_{n} \geq 0\right\} \text {. }
$$

Remark 2.2. For each fixed value of $n \in \mathbb{N}_{2}, z \in U$, the following real-valued functions

$$
F(f)=\left|a_{n}\right|, \quad F(f)=\left|b_{n}\right|, \quad F(f)=|f(z)|, \quad F(f)=\left|W_{\alpha, \beta}^{k, q} f(z)\right| \quad(f \in \mathcal{H})
$$

are continuous and convex on $\mathcal{H}$.

Also, for $\gamma \geq 0,0<r<1$, the real-valued functional

$$
F(f)=\left(\frac{1}{2 \pi} \int_{0}^{2 \pi}\left|f\left(r e^{i \theta}\right)\right|^{\gamma} d \theta\right)^{\frac{1}{\gamma}} \quad(f \in \mathcal{H})
$$

is continuous and convex on $\mathcal{H}$.

By making use of Theorem 2.4 and Lemma 1.1, we obtain the following corollaries:

Corollary 2.2. Let $f \in W T_{\mathcal{H}}(\rho, \tau, \alpha, \beta, k, q),|z|=r<1$. Then

$$
r+\frac{1-\rho}{2(2-\rho+\tau)\left|\Phi_{2}(k, \alpha, \beta)\right|^{q}} r^{2} \leq|f(z)| \leq r+\frac{1-\rho}{2(2-\rho+\tau)\left|\Phi_{2}(k, \alpha, \beta)\right|^{q}} r^{2}
$$

and

$$
r+\frac{1-\rho}{2(2-\rho+\tau)} r^{2} \leq\left|W_{\alpha, \beta}^{k, q} f(z)\right| \leq r+\frac{1-\rho}{2(2-\rho+\tau)} r^{2} .
$$

The result is sharp. The function $h_{n}$ of the form (2.7) is the extremal function. 
Corollary 2.3. Let $\gamma \geq 0,0<r<1$. If $f \in W T_{\mathcal{H}}(\rho, \tau, \alpha, \beta, k, q)$, then

$$
\frac{1}{2 \pi} \int_{0}^{2 \pi}\left|f\left(r e^{i \theta}\right)\right|^{\gamma} d \theta \leq \frac{1}{2 \pi} \int_{0}^{2 \pi}\left|h_{2}\left(r e^{i \theta}\right)\right|^{\gamma} d \theta
$$

and

$$
\frac{1}{2 \pi} \int_{0}^{2 \pi}\left|z f^{\prime}\left(r e^{i \theta}\right)\right|^{\gamma} d \theta \leq \frac{1}{2 \pi} \int_{0}^{2 \pi}\left|z h_{2}^{\prime}\left(r e^{i \theta}\right)\right|^{\gamma} d \theta .
$$

The function $h_{2}$ is the function defined by (2.7).

\section{Conclusion}

The results we obtained in this paper which may be considered as a useful tool for those who are interested in the above-mentioned topics for further research. It may also be used to find prospective applications in some areas of mathematics and physics.

\section{References}

[1] J. W. Alexander, Functions which map the interior of the unit circle upon simple region, Ann. of Math. 17(1) (1915), 12-22. https://doi.org/10.2307/2007212

[2] F. M. Al-Oboudi, On univalent functions defined by a generalized Salagean operator, Int. J. Math. Math. Sci. 27 (2004), 1429-1436. https://doi.org/10.1155/S0161171204108090

[3] S. D. Bernardi, Convex and starlike univalent functions, Trans. Amer. Math. Soc. 135 (1969), 429-446. https://doi.org/10.1090/S0002-9947-1969-0232920-2

[4] N. E. Cho and H. M. Srivastava, Argument estimates of certain analytic functions defined by a class of multiplier transformations, Math. Comput. Modeling 37(1-2) (2003), 39-49. https://doi.org/10.1016/S0895-7177(03)80004-3

[5] N. E. Cho and T. H. Kim, Multiplier transformations and strongly close-to-convex functions, Bull. Korean Math. Soc. 40(3) (2003), 399-410. https://doi.org/10.4134/BKMS.2003.40.3.399

[6] J. Clunie and T. Sheil-Small, Harmonic univalent functions, Ann. Acad. Aci. Fenn. Ser. A I Math. 9 (1984), 3-25. https://doi.org/10.5186/aasfm.1984.0905

[7] J. Dziok, On Janowski harmonic functions, J. Appl. Anal. 21(2) (2015), 99-107. https://doi.org/10.1515/jaa-2015-0010

[8] I. B. Jung, Y. C. Kim and H. M. Srivastava, The Hardy space of analytic functions 
associated with certain one-parameter families of integral operators, J. Math. Anal. Appl. 176 (1993), 138-147. https://doi.org/10.1006/jmaa.1993.1204

[9] P. Montel, Sur les familles de fonctions analytiques qui admettent des valeurs exceptionnelles dans un domaine, Ann. Sci. Ecole Norm. Sup. 29 (1912), 487-535. https://doi.org/10.24033/asens.652

[10] G. S. Salagean, Subclasses of univalent functions, Lecture Notes in Math., Springer Verlag, Berlin 1013 (1983), 362-372. https://doi.org/10.1007/BFb0066543

[11] T. Sheil-Small, Constants for planer Harmonic mappings, J. London Math. Soc. 42(2) (1990), 237-248. https://doi.org/10.1112/jlms/s2-42.2.237

[12] H. M. Srivastava and A. A. Attiya, An integral operator associated with the HurwitzLerch Zeta function and differential subordination, Integral Transforms and Special Functions 18(3) (2007), 207-216. https://doi.org/10.1080/10652460701208577

[13] S. R. Swamy, Inclusion properties of certain subclasses of analytic functions, Int. Math. Forum 7(36) (2012), 1751-1760.

[14] B. A. Uralegaddi and C. Somanatha, Certain classes of univalent functions, in: Current Topics in Analytic Function Theory, (Edited by H. M. Srivastava and S. Own), 371-374, World Scientific, Singapore, 1992. https://doi.org/10.1142/9789814355896_0032

[15] A. K. Wanas, New differential operator for holomorphic functions, Earthline J. Math. Sci. 2(2) (2019), 527-537. https://doi.org/10.34198/ejms.2219.527537

[16] A. K. Wanas and G. Murugusundaramoorthy, Differential sandwich results for Wanas operator of analytic functions, Mathematica Moravica 24(1) (2020), 17-28. https://doi.org/10.5937/MatMor2001017K

Abbas Kareem Wanas

Department of Mathematics

College of Science

University of Al-Qadisiyah, Iraq

e-mail: abbas.kareem.w@qu.edu.iq

This is an open access article distributed under the terms of the Creative Commons Attribution License (http://creativecommons.org/licenses/by/4.0/), which permits unrestricted, use, distribution and reproduction in any medium, or format for any purpose, even commercially provided the work is properly cited.. 